\title{
Investigating the Design of Condition Monitoring Systems to Evaluate Surface Roughness under the Variability in Tool wear and Fixturing Conditions
}

\author{
Jabbar Abbas ${ }^{\mathrm{a}}$, Amin Al-Habaibeh ${ }^{\mathrm{b}}$, Daizhong Su \\ Advanced Design and Manufacturing Engineering Centre-ADMEC \\ School of Architecture, Design and the Built Environment \\ Nottingham Trent University, Nottingham, NG1 4BU, UK. \\ ajabbar.abbas@ntu.ac.uK, ${ }^{\mathrm{b}} \mathrm{Amin} . \mathrm{Al}-\mathrm{Habaibeh@ntu.ac.uk,}{ }^{\mathrm{c}}$ daizhong.su@ntu.ac.uk
}

Keywords: Surface Roughness, Fixturing, Monitoring System, Elastic Materials.

\begin{abstract}
Surface finish of machined parts in end milling operations is significantly influenced by process faults such as tool wear and tool holding (fixturing system). Therefore, monitoring these faults is considerably important to improve the quality of the product. In this paper, an investigation is presented to design the condition monitoring system to evaluate the surface roughness of the workpiece under effects of gradual tool wear and different types of the fixturing system. Automated Sensor and Signal Processing Selection (ASPS) approach is implemented and tested to determine the sensitivity of the sensory signals to estimate surface roughness under the variable conditions in comparison to surface roughness measurement device. The results indicate that the system is capable of detection the change and the trend in surface roughness. However, the sensitive features are found to be different based on the change in the fixturing system.
\end{abstract}

\section{Introduction}

Surface roughness is considered as one of the most important parameters to determine the quality of machined parts. Surface roughness is defined as a group of irregular waves in the surface, measured in micrometers $(\mu \mathrm{m})$ and mostly in the industry represent as roughness average (Ra) [1]. Practically, many influences which usually effect on surface roughness including vibration and inaccuracies in the machine tool, the runout errors of the tools [2]. These errors generally obtained from the tool wear or imperfect fixturing system. Fixtures are essential devices for locating, clamping and supporting precisely a work piece or a cutting tool in a given orientation and position. When the contact between the mating surfaces is changed the fixturing stability will also change [3]. Therefore it is important to monitor and control machined surface quality. Generally, on-line monitoring system consists of sensors, signal processing stages, and decision making systems to interpret the sensory information. Recently, monitoring surface roughness is performed by using cutting force signals [4]. All the above issues need to be investigated with regard to the effect of the change of cutting tool and fixturing conditions on the surface roughness under the observing of the monitoring system. The hypothesis is that design a condition monitoring system to evaluate the workpiece roughness under the variation in the tool condition and fixturing stability.

\section{Implemented Methodology}

The methodology of the paper consists three steps aim to measure the correlation between the sensitivity and Ra values automatically as explained in the following steps:

1. Linear Regression (LR) method: This method is a statistical analysis assessing the association between two variables. The line is obtained by using the least squares straight line fitting. The slop of the line regression is defined as:

$$
\text { slop }=\frac{N \sum_{i=1}^{N} x_{i} y_{i}-\left(\sum_{i=1}^{N} x_{i}\right)\left(\sum_{i=1}^{N} y_{i}\right)}{N \sum_{i=1}^{n} y^{2}{ }_{i 1}-\left(\sum_{i=1}^{N} x_{i}\right)}
$$


Where $N$ : Number of values or elements; $\mathrm{x}$ and $\mathrm{y}$ are the variables.

2. Surface roughness measurement: By using the roughness tester, it is possible to track the machining process and take the real measurement of the surface, this search is to find the relationship between the sensory data from indirect measurement with the actual roughness.

3. Correlation Coefficient: The correlation coefficient is a quantity that gives the quality of a least squares fitting to the original data or to define the relation between two cases. In the current paper, this coefficient will be used to find the relation between the sensitivity of the monitoring sensor and the surface roughness of the workpiece.

\section{Performed Experiment}

As illustrated in Fig 1, several sensory signals are used in this study including cutting forces (Fx, Fy and $\mathrm{Fz}$ ) using 3-component dynamometer (Kistler 9257A), strain, accelerometer (vibration), Acoustic emission (AE), eddy current, power sensor, and microphone. The dynamic and quasi static force signals are monitored using a strain sensor (Kistler 9232A). Both the force dynamometer and the strain sensor are connected to a 4-channel charge amplifier (Kistler 5070A). The AE sensor (Kistler 8152b111) is attached to the workpiece and connected to AE coupler (Kistler 5125B). The accelerometers (B\&K4366, Kistler8707B) are connected to charge amplifier (Kistler 5001) and coupler (5134B) respectively. Sound signals are collected using a microphone (Mic-EM400). Eddy current sensors (Edx, Edy -IC12-02) and Power sensor (Pwr-IP-151) are connected directly to the data acquisition card NI PCI-6071E. Mitutoya apparatus (SJ-210) is used for the surface roughness measurements. The experiments performed on CNC milling machine using Aluminium workpiece.

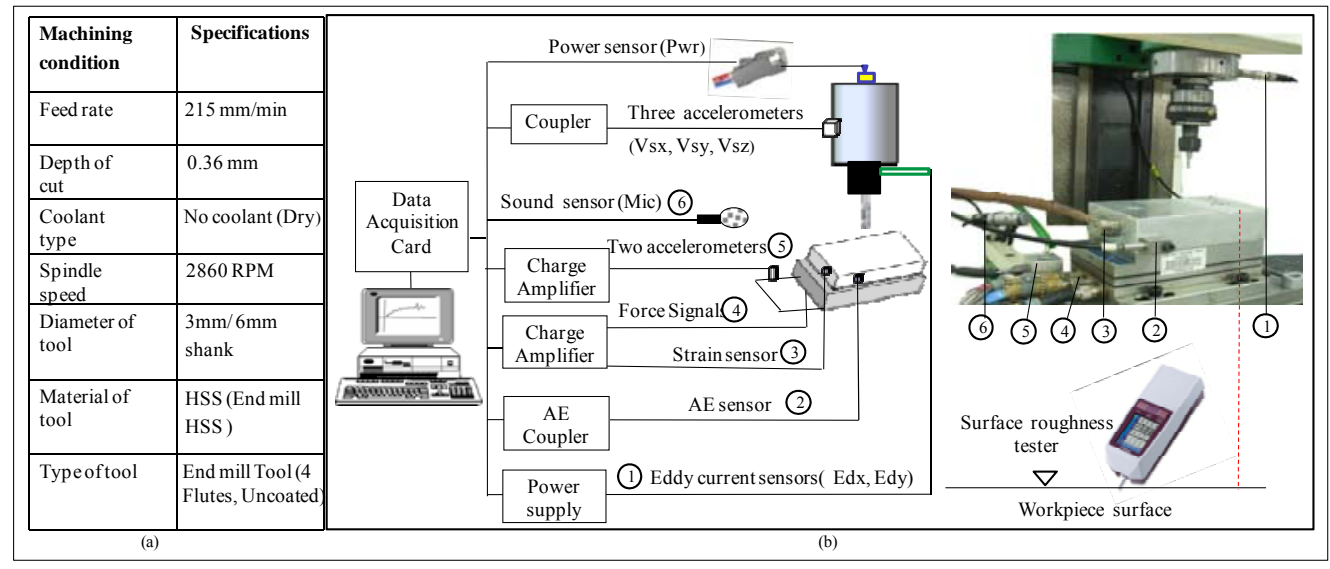

Fig 1: a. Table of The machining parameters. b. Schematic diagram of experimental setup.

\section{Experimental Results and Discussion}

To emulate a fixturing system with low rigidity, the shank of the tool is covered by three different materials namely rubber, copper and aluminium with thickness of $1 \mathrm{~mm}$ as shown in Fig $2 \mathrm{a}$. The raw signals for the tools are collected from the sensors to monitor 27 machining runs/samples for each type of tools. The relation between the surface roughness and the sensitivity of the sensory signals under the effect of the changing the quality of the fixturing system as follows:

1. The sensitivity of condition monitoring system. This research builds on the Automated Sensor and Signal Processing Selection (ASPS) approach [5] to investigate further combination of techniques and parameters using a wide range of signal analysis and simplification techniques. The proposed monitoring system consists simplification the collected signals and measuring the sensitivity of the sensors. The raw signals are processed using several time domain signal processing methods to extract the Sensory Characteristic Features (SCFs). The signal processing methods used are maximum $(\max )$, minimum $(\min )$, standard deviations $(s t d)$, the average $(\mu)$, the range, the skew, kurtosis value $(K)$ and power. The SCFs are arranged according to their 
sensitivities to tool wear based on the absolute slope of the linear regression method as shown in Fig $2 b$. Fig $2 b$ presents examples of high and low-sensitivity SCFs to tool wear.

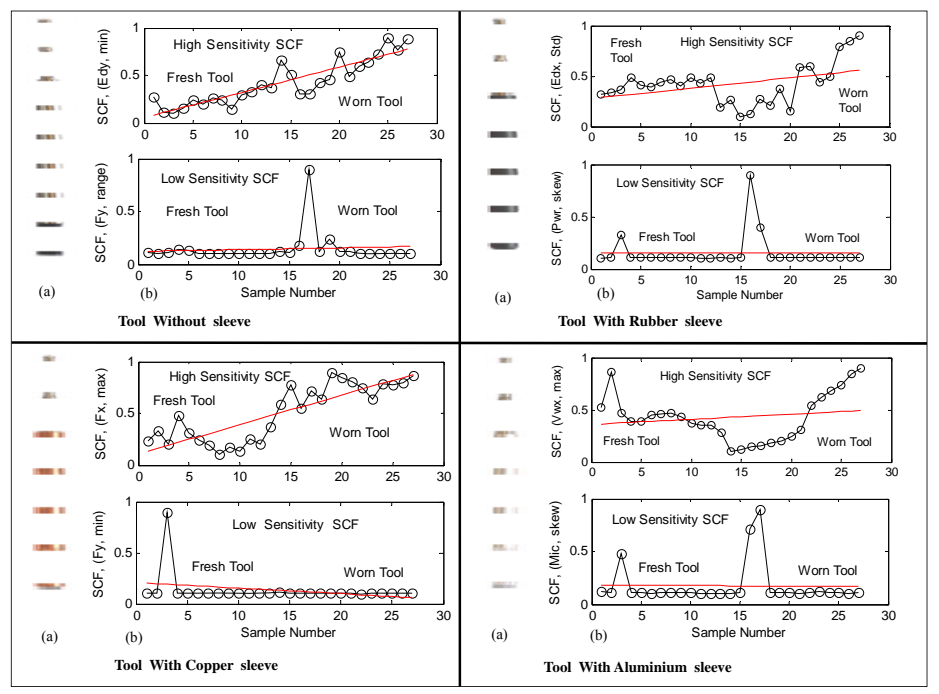

Fig 2: (a) Cutting tools, (b) Example of low, medium and high sensitivity SCF for the tools.

The SCFs are visually inspected and it has been found that SCFs with high absolute slope show higher sensitivity to the fault. Fig 2 prove that the change in the characteristic of the fixturing system has caused change in the most sensitive sensors and signal processing systems that can be used to detect tool wear. For example, with normal fixturing system, eddy current signals (Edy) and sound are found to be the most sensitive to detect tool wear. However, with the rubber sleeve system, eddy current (Edx) and force signals are found to be the most sensitive signals to detect tool wear. Differently, with holding by copper sleeve, force and power signals are indicated more performance to sense the tool conditions. Similarly, with clamping by the aluminum sleeve, Vibration signals (Vwx, Vwy) have effectively stated the tool wear.

2. Surface roughness of machined surface. Surface specification can also be a good reference point in determining the stability of a production process, because the stability of the machine is contingent on the quality of the operating part. The surface roughness of the workpiece has been measured for each track of 27 tests with used normal fixturing and different elastic material sleeves namely rubber, copper and aluminum. Fig 3 has shown the results of the surface roughness for four cases. The consequences of the patterns indicate that the values of the surface roughness when using tool without sleeve is less than those obtained when using tools with rubber, copper and aluminum sleeves. The reason for these results may be the difference in the modulus of elasticity of sleeve material.

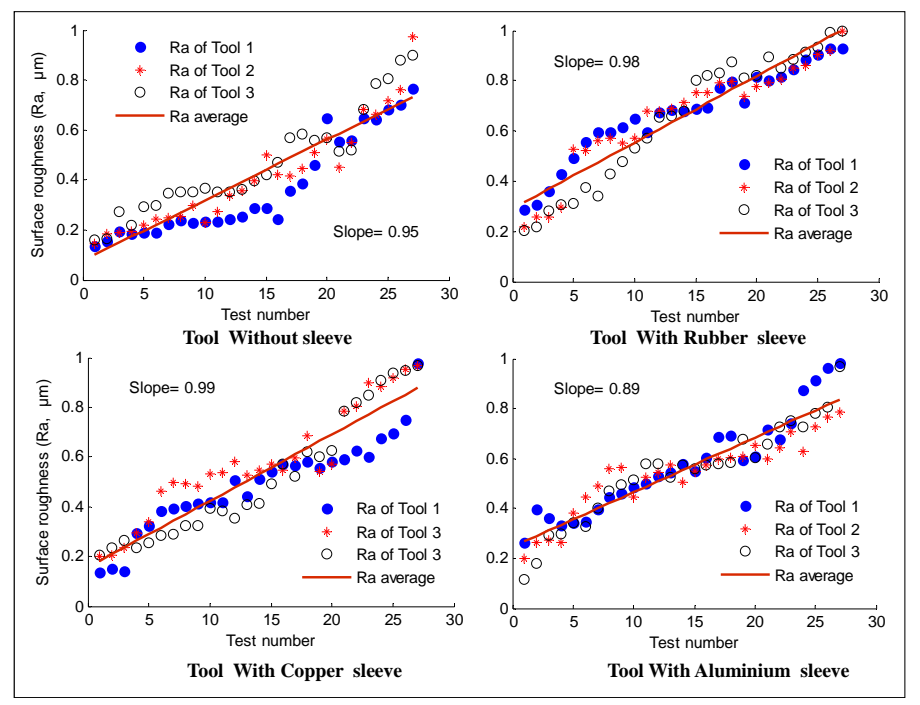

Fig 3: Surface roughness of workpiece for four types of fixturing materials. 
3. The correlation coefficient between the sensitivity SCF and surface roughness. In Fig 4, high and low correlation have been illustrated as example of correlation according to the relation between the sensor features and surface roughness for the normal tool and tool with rubber, copper and aluminum sleeve respectively. It is clear that the correlation between two techniques (i.e. sensor characteristic features (SCF) and surface roughness) has achieved high agreement especially in the maximum values of the sensitivity.

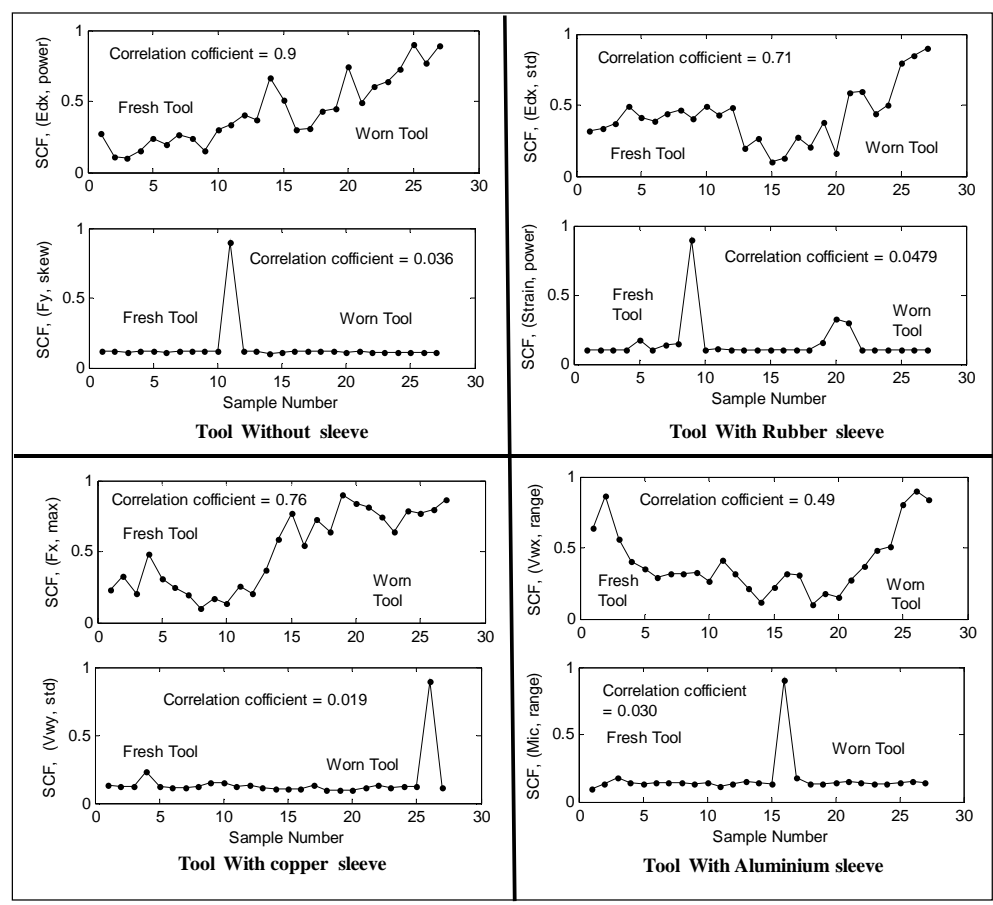

\section{Conclusion}

Fig 4: Example of low, medium and high sensitivity SCF for the tools.

This paper has investigated the relation between the surface roughness of the workpiece and the sensitivity of the monitoring system which designed to detect any abnormalities of the tool wear and the stability of the fixturing system. Automated Sensor and Signal Processing Selection (ASPS) approach [5] has been implemented to define the most sensitive features (SCF) which are constructed from the sensor and the signal processing method. Linear regression slop method used to measure the sensitivity of the signal, then these values combined with the real roughness measurements using surface tester. The result proves the capability of the designed monitoring that sensor sensitivity are significantly effected by the increasing the surface roughness and could be created a higher correlation. In addition, it is dictated that the poor quality of surface obtained from the tool wear and the instability of the fixturing system.

\section{References}

[1] Oguz Colak, Cahit Kurbanoglu, and M. Cengiz Kayacan, in: Milling surface roughness prediction using evolutionary programming methods, Materials and Design, Vol. 28, Issue 2, (2007), p. 657-666.

[2] Vikas Upadhyay, P.K. Jain, N.K. Mehta, in: In-process prediction of surface roughness in turning of Ti-6Al-4V alloy using cutting parameters and vibration signals, Measurement, Vol. 46, Issue 1, (2013), p. 154-160.

[3] Preben Hansen, in: Solid contact, the advantages of dual and triple contact dual and triple contact tool holding systems for high speed machining, Vol. 53 , No. 7. (2001).

[4] J. Abbas, A. Al-Habaibeh and D. SU, in: The Investigation of prediction surface roughness from machining forces in end milling processes, Key Eng. Materials. Vol 486, (2011), p 91-94.

[5] A. Al-Habaibeh, Abd Al-Azmi, N. Radwan and Yang Song, in: The Application of Force and Acoustic Emission Sensors for Detecting Tool Damage in Turning Processes, Key Engineering Materials, Vols. 419-420, , (2010), p. 381-384. 